Case Report

\title{
17-Year-Old Boy with Renal Failure and the Highest Reported Creatinine in Pediatric Literature
}

\author{
Vimal Master Sankar Raj, ${ }^{1}$ Jessica Garcia, ${ }^{2}$ and Roberto Gordillo ${ }^{1}$ \\ ${ }^{1}$ Department of Pediatric Nephrology, University of Illinois College of Medicine at Peoria (UICOMP), Peoria, IL 61603, USA \\ ${ }^{2}$ Department of Pediatrics, University of Illinois College of Medicine at Peoria (UICOMP), Peoria, IL 61603, USA
}

Correspondence should be addressed to Vimal Master Sankar Raj; vraj@uicomp.uic.edu

Received 30 April 2015; Accepted 1 June 2015

Academic Editor: Giovanni Montini

Copyright (c) 2015 Vimal Master Sankar Raj et al. This is an open access article distributed under the Creative Commons Attribution License, which permits unrestricted use, distribution, and reproduction in any medium, provided the original work is properly cited.

The prevalence of chronic kidney disease (CKD) is on the rise and constitutes a major health burden across the world. Clinical presentations in early CKD are usually subtle. Awareness of the risk factors for CKD is important for early diagnosis and treatment to slow the progression of disease. We present a case report of a 17-year-old African American male who presented in a life threatening hypertensive emergency with renal failure and the highest reported serum creatinine in a pediatric patient. A brief discussion on CKD criteria, complications, and potential red flags for screening strategies is provided.

\section{Background}

Prevalence of chronic kidney disease (CKD) is increasing significantly and it has poor outcomes if not diagnosed and treated early in its course [1]. CKD is a public health issue that affects 9 to $12 \%$ of the population in the USA $[2,3]$. When management is early and adequate, the rate of progression to kidney failure can be slowed, comorbidities prevented, and the morbidity and mortality of cardiovascular disease associated with CKD decreased. There is lack of information on incidence and prevalence of earlier stages of CKD in children, as most of these patients are asymptomatic [4].

The National Kidney Foundation (NKF) created the Kidney Disease Outcomes Quality Initiative (KDOQI) guidelines and definition of CKD to facilitate the diagnosis and management by primary care physicians. Serum creatinine, a product of muscle metabolism, has been widely used as a marker for glomerular filtration rate (GFR). We describe a clinical case of an adolescent male with the highest serum creatinine reported in a pediatric patient. The primary aim of this case report was to create awareness of chronic kidney disease among general practitioners and to stress that clinical manifestations could be subtle in the early stages of the disease.

\section{Case Presentation}

A 17-year-old African American male who was previously healthy with the exception of high blood pressure presented to a referring hospital with a 4-day history of coughing, vomiting, headache, facial edema, and lower extremity cramping. One day prior to admission, he also had noticed decreased urine output. At the referring hospital, he was found to be hypertensive with a serum creatinine of $52 \mathrm{mg} / \mathrm{dL}$ $(4597 \mu \mathrm{mol} / \mathrm{L})$ and was transferred to our pediatric intensive care unit for further evaluation and treatment.

Patient was born at term gestation and there were no significant health problems. Patient during his routine clinic visits was noted to have high blood pressure by his primary care physician but no further evaluation was done as the blood pressure was attributed to his obesity. Patient did not have any prior surgeries and was not taking any medications. There was no significant history of renal disease, dialysis, or kidney transplant in any of the family members.

On admission, patient was noted to be afebrile, hypertensive with blood pressure of 179/93 $\mathrm{mm} \mathrm{Hg}$ and heart rate of 88 beats per minute, and tachypneic with respiratory rate in the 40 s but saturating at $100 \%$ on room air. His anthropometric measurements showed his height to be 
$180 \mathrm{~cm}$, his weight to be $115 \mathrm{~kg}$, and body mass index to be $>99 \%$. Pertinent positives in physical exam include obesity, respiratory distress with nasal flaring, bilateral periorbital edema, and bilateral lower extremity edema. Laboratory results showed the patient to have azotemia with blood urea nitrogen (BUN) of $203 \mathrm{mg} / \mathrm{dL}$ and serum creatinine of $52 \mathrm{mg} / \mathrm{dL}$, measured by enzymatic method. He had severe metabolic acidosis with bicarbonate of $10 \mathrm{mmol} / \mathrm{L}$. Patient also had severe electrolyte imbalances including hyponatremia $(126 \mathrm{mmol} / \mathrm{L})$, hyperkalemia $(6.3 \mathrm{mmol} / \mathrm{L})$, hyperphosphatemia $(10.5 \mathrm{mg} / \mathrm{dL})$, hypocalcemia $(5.3 \mathrm{mg} / \mathrm{dL})$, and severe anemia with hemoglobin at $5.1 \mathrm{~g} / \mathrm{dL}$. Parathyroid hormone levels were elevated at $682 \mathrm{pg} / \mathrm{mL}$ (normal 11$80 \mathrm{pg} / \mathrm{mL}$ ). Urine dipstick showed $3+$ protein and $2+$ blood and a urine protein to creatinine ratio was elevated at 14 (normal <0.2). Serological workup including complement levels, antinuclear antibodies, antinuclear cytoplasmic antibodies, and antiphospholipid antibodies was negative. Chest X-ray was significant for cardiomegaly and pulmonary edema. Echocardiogram showed severe left ventricular hypertrophy. Renal ultrasound revealed bilateral small, echogenic kidneys (right kidney $7.7 \times 5.2 \times 4.8 \mathrm{~cm}$; left kidney $8.7 \times 4.3 \times$ $4.1 \mathrm{~cm}$ ) consistent with dysplastic kidneys. Patient emergently underwent hemodialysis for fluid overload and to correct electrolyte imbalance. His anemia and electrolyte imbalances were slowly corrected. He is currently transitioned to peritoneal dialysis awaiting kidney transplant.

\section{Discussion}

CKD is a heterogeneous group of diseases with altered structure and function of the kidneys with varying manifestation based on underlying etiology. Based on KDIGO guidelines, CKD is classified into various stages using GFR and the degree of albuminuria (Tables 1(a) and 1(b)).

The modified Schwartz formula used to calculate GFR is derived from the CKiD (chronic kidney disease in children) [5] and uses the following formula:

eGFR $=K$ (height in centimeters)/Serum creatinine with the constant $K$ value being 0.413 .

GFR varies with age, gender, and body size and increases from infancy to adulthood. Normative data of GFR based on age is presented in Table 2 . This needs to be taken into consideration on CKD classification in pediatric population.

\section{Epidemiology}

Most epidemiological information on adult CKD is available from data on ESRD patients [6]. This represents only the tip of the iceberg and the actual incidence of early stage CKD will be much higher. The exact prevalence of childhood CKD is unknown but it has been estimated at 82 cases per million with ESRD around 15 cases per million based on national registries [7-9]. Though pediatric ESRD contributes only $2 \%$ to the total ESRD burden, the mortality rate in adolescents is about 30 to $150 \%$ higher compared to the general population $[8,10]$ indicating the need for specialized
TABLE 1: (a) CKD based on GFR [14]. (b) Persistent albuminuria and risk for CKD [14].

(a)

\begin{tabular}{llc}
\hline $\begin{array}{l}\text { GFR } \\
\text { categories }\end{array}$ & Description & $\begin{array}{c}\text { Range } \\
\left(\mathrm{mL} / \mathrm{min} / 1.73 \mathrm{~m}^{2}\right)\end{array}$ \\
\hline G1 & Normal or high & $\geq 90$ \\
\hline G2 & Mildly decreased & $60-89$ \\
\hline G3a & $\begin{array}{l}\text { Mildly to moderately } \\
\text { decreased }\end{array}$ & $45-59$ \\
\hline G3b & $\begin{array}{l}\text { Moderately to severely } \\
\text { decreased }\end{array}$ & $30-44$ \\
\hline G4 & Severely decreased & $15-29$ \\
\hline G5 & Kidney failure & $<15$ \\
\hline
\end{tabular}

(b)

\begin{tabular}{llc}
\hline Categories & Description & Range \\
\hline A1 & Normally to mildly increased & $<30 \mathrm{mg} / \mathrm{g}$ \\
\hline A2 & Moderately increased & $\begin{array}{c}<3 \mathrm{mg} / \mathrm{mmol} \\
30-300 \mathrm{mg} / \mathrm{g}\end{array}$ \\
\hline A3 & Severely increased & $3-30 \mathrm{mg} / \mathrm{mmol}$ \\
& & $>300 \mathrm{mg} / \mathrm{g}$ \\
\hline
\end{tabular}

Adapted from KDIGO 2012.

TABLE 2: Normal glomerular filtration rate (GFR) in children and adolescents [15].

\begin{tabular}{lc}
\hline Age & Mean GFR $\pm \mathrm{SD}\left(\mathrm{mL} / \mathrm{min} / 1.73 \mathrm{~m}^{2}\right)$ \\
\hline 1 week (males and females) & $41 \pm 15$ \\
2-8 weeks (males and females) & $66 \pm 25$ \\
$>8$ weeks (males and females) & $96 \pm 22$ \\
2-12 years (males and females) & $133 \pm 27$ \\
$13-21$ years (males) & $140 \pm 30$ \\
$13-21$ years (females) & $126 \pm 22$ \\
\hline
\end{tabular}

Adapted from National Kidney Foundation.

care. The expected remaining life time of children below 14 years of age with ESRD and on dialysis calculated per US renal data system (USRDS) [8] is only 20 years. Hence, the importance of primary prevention with early detection and aggressive intervention cannot be overstated.

\section{Etiology}

The etiology for ESRD varies with age. Structural anomalies contribute to a large degree to ESRD in younger children, while it is predominantly glomerular diseases in older children. As per NAPRTCS 2011 review data, about $14.2 \%$ of all pediatric dialysis patients had ESRD secondary to hypoplastic/dysplastic kidney with obstructive uropathy at $12.6 \%$ (Table 3).

\section{Screening Strategies for CKD}

Screening strategies are aimed at early detection and intervention for CKD so as to slow the progression of disease. 
TABLE 3: Pediatric dialysis patient demographics [16].

\begin{tabular}{|c|c|c|}
\hline \multirow{2}{*}{ All dialysis patients } & $N$ & $\%$ \\
\hline & 7039 & 100.0 \\
\hline \multicolumn{3}{|l|}{ Primary diagnosis } \\
\hline FSGS & 1016 & 14.4 \\
\hline Hypoplastic/dysplastic kidney & 998 & 14.2 \\
\hline Obstructive uropathy & 888 & 12.6 \\
\hline Reflux nephropathy & 244 & 3.5 \\
\hline SLE nephritis & 226 & 3.2 \\
\hline HUS & 216 & 3.1 \\
\hline Chronic GN & 214 & 3.0 \\
\hline Polycystic disease & 201 & 2.9 \\
\hline Congenital nephrotic syndrome & 182 & 2.6 \\
\hline Prune belly & 144 & 2.0 \\
\hline Medullary cystic disease & 140 & 2.0 \\
\hline Idiopathic crescentic GN & 130 & 1.8 \\
\hline Familial nephritis & 130 & 1.8 \\
\hline MPGN-type I & 116 & 1.6 \\
\hline Pyelonephritis/interstitial nephritis & 101 & 1.4 \\
\hline Cystinosis & 99 & 1.4 \\
\hline Renal infarct & 90 & 1.3 \\
\hline Berger's (IgA) nephritis & 86 & 1.2 \\
\hline Henoch-Schönlein nephritis & 67 & 1.0 \\
\hline MPGN-type II & 64 & 0.9 \\
\hline Wilms' tumor & 55 & 0.8 \\
\hline Wegener's granulomatosis & 49 & 0.7 \\
\hline Drash syndrome & 39 & 0.6 \\
\hline Other systemic immunologic diseases & 37 & 0.5 \\
\hline Oxalosis & 32 & 0.5 \\
\hline Membranous nephropathy & 29 & 0.4 \\
\hline Sickle cell nephropathy & 21 & 0.3 \\
\hline Diabetic GN & 10 & 0.1 \\
\hline Other & 887 & 12.6 \\
\hline Unknown & 528 & 7.5 \\
\hline
\end{tabular}

Adapted from NAPRTCS 2011.

Though routine urine screening for CKD has not been found to be cost effective in the general population and has not been recommended by AAP, it is important to identify and screen children at high risk. Some of the high risk populations at risk for $\mathrm{CKD}$ are mentioned below:

(i) Prematurity and being small for gestational age.

(ii) Congenital abnormalities of the kidney and urinary tract.

(iii) H/o poor growth or failure to thrive.

(iv) Family history of kidney diseases and relatives on dialysis or transplant.

(v) Electrolyte or acid-base abnormalities.

(vi) Body mass index (BMI) > 95th percentile.

(vii) Blood pressure greater than the 95\% recorded on multiple visits. (viii) Polyuria or inappropriately dilute urine.

(ix) Gross hematuria.

(x) Dysfunctional voiding, urinary incontinence, or prolonged enuresis.

(xi) H/o recurrent UTI.

A thorough history and physical examination during well child visits could help us in identifying these children with high risk for kidney disease. Once identified, these children should have their urine checked for proteinuria, their renal function analyzed by measuring creatinine, and their blood pressure regularly screened. Children with evidence of kidney damage should be sent to a specialist for further investigation and treatment. For our patient, blood pressure $>95 \%$ and the BMI $>95 \%$ should have prompted a screening for potential kidney disease.

\section{CKD Complications}

Cardiovascular disease accounts for most deaths in pediatric CKD similar to adult onset CKD. In contrast to adult CKD patients, where atherosclerosis and coronary vascular disease are much more common, arrhythmias account for the majority of cardiovascular death in children (19.6\%) [11]. Traditional risk factors for CVD such as dyslipidemia and hypertension along with nontraditional risk factors such as anemia, disorders of calcium phosphorus metabolism, and increased chronic inflammation are highly prevalent in CKD population. These also contribute significantly to the cardiovascular burden in these children.

Anemia is a common complication in CKD secondary to impaired erythropoiesis. As CKD progresses, so does the prevalence of anemia in these children. Factors such as malnutrition, blood loss, iron deficiency, inadequate dialysis, and uncontrolled secondary hyperparathyroidism should always be kept in mind while managing resistant anemia. KDIGO recommends maintaining hemoglobin levels between 10 and $12 \mathrm{~g} / \mathrm{dL}$ to reduce need for transfusion. Iron levels should be checked and adequately supplemented in all CKD patients before initiating or increasing dose of erythropoiesis stimulating agents.

Metabolic bone disorder (CKD-MBD) is a major complication in CKD. This occurs secondary to the inability of kidneys to excrete phosphorus and synthesize active vitamin D. Net result is secondary hyperparathyroidism. Disorders in calcium phosphorus balance and secondary hyperparathyroidism play a major role in vascular calcification in CKD and subsequent cardiovascular mortality and morbidity.

Fluid and electrolyte imbalances are especially common in children with $\mathrm{CKD}$ secondary to congenital anomalies of the kidneys and urinary tract (CAKUT). In CAKUT impaired urinary concentrating ability could present with polyuria and can present with dehydration. Disorder of sodium, potassium, and acid-base balance is also very common in these children due to impaired tubular handling. As the normal tubular response to $\mathrm{ADH}$ is not present, special attention should be paid to the fluid and electrolyte replacement in these children in the setting of dehydration. 
Growth failure and neurocognitive delay are important concerns for young children with CKD $[12,13]$. Providing adequate calories and protein intake are important in children with CKD as they are more prone to muscle wasting and anorexia. Factors such as systemic inflammation, oral aversion, and alteration in hormonal levels or resistance to action of hormones (follicle stimulating hormone, luteinizing hormone, growth hormone, and thyroid hormone) also contribute to short stature and CKD in children. In infants and toddlers with CKD to maximize nutritional intake a gastrostomy tube is often placed to provide adequate calories and fluids. Optimal nutrition and use of growth hormone replacement are often needed in children with CKD to ensure that they reach their growth potential.

\section{Conclusion}

Our patient had bilateral dysplastic kidneys and ultimately progressed to ESRD. Though he had potential red flags including obesity and high blood pressure noted on multiple occasions, the thought of kidney disease was not entertained. A screening urine dipstick could have prevented this life threatening admission with hypertensive emergency, severe anemia, and multiple electrolyte imbalances. Earlier detection of kidney disease and control of hypertension and proteinuria could have slowed the progression of disease and also would have allowed the time to plan for his renal replacement therapy in a safe manner. Chronic kidney disease is a growing health burden and awareness of it among primary care physicians is essential in early diagnosis and treatment.

\section{Abbreviations}

CKD: Chronic kidney disease

ESRD: End stage renal disease

GFR: Glomerular filtration rate

BUN: Blood urea nitrogen.

\section{Disclosure}

The authors have no financial relationships relevant to this paper to disclose.

\section{Conflict of Interests}

The authors declare that there is no conflict of interests to disclose.

\section{References}

[1] V. Agrawal, A. K. Ghosh, M. A. Barnes, and P. A. McCullough, "Perception of indications for nephrology referral among internal medicine residents: a national online survey," Clinical Journal of the American Society of Nephrology, vol. 4, no. 2, pp. 323-328, 2009.

[2] J. Coresh, D. Byrd-Holt, B. C. Astor et al., "Chronic kidney disease awareness, prevalence, and trends among U.S. adults,
1999 to 2000," Journal of the American Society of Nephrology, vol. 16, no. 1, pp. 180-188, 2005.

[3] J. Coresh, E. Selvin, L. A. Stevens et al., "Prevalence of chronic kidney disease in the United States," Journal of the American Medical Association, vol. 298, no. 17, pp. 2038-2047, 2007.

[4] B. A. Warady and V. Chadha, "Chronic kidney disease in children: the global perspective," Pediatric Nephrology, vol. 22, no. 12, pp. 1999-2009, 2007.

[5] L. Copelovitch, B. A. Warady, and S. L. Furth, "Insights from the chronic kidney disease in children (CKiD) study," Clinical Journal of the American Society of Nephrology, vol. 6, no. 8, pp. 2047-2053, 2011.

[6] J. Coresh, B. C. Astor, T. Greene, G. Eknoyan, and A. S. Levey, "Prevalence of chronic kidney disease and decreased kidney function in the adult US population: third National Health and Nutrition Examination Survey," The American Journal of Kidney Diseases, vol. 41, no. 1, pp. 1-12, 2003.

[7] S. F. Massengill and M. Ferris, "Chronic kidney disease in children and adolescents," Pediatrics in Review, vol. 35, no. 1, pp. 16-29, 2014.

[8] U.S. Renal Data System (USRDS), Annual Data Report: Atlas of End-Stage Renal Disease in the United States, National Institutes of Health, National Institute of Diabetes and Digestive and Kidney Diseases, Bethesda, Md, USA, 2004.

[9] Clinical Coordinating Center and Data Coordinating Center, North American Pediatric Renal Transplant Cooperative Study (NAPRTCS), Novartis Pharmaceutical, 2005.

[10] S. P. McDonald and J. C. Craig, "Long-term survival of children with end-stage renal disease," The New England Journal of Medicine, vol. 350, no. 26, pp. 2654-2662, 2004.

[11] R. Shroff, D. J. Weaver Jr., and M. M. Mitsnefes, "Cardiovascular complications in children with chronic kidney disease," Nature Reviews Nephrology, vol. 7, no. 11, pp. 642-649, 2011.

[12] R. H. Mak, W. W. Cheung, J.-Y. Zhan, Q. Shen, and B. J. Foster, "Cachexia and protein-energy wasting in children with chronic kidney disease," Pediatric Nephrology, vol. 27, no. 2, pp. 173-181, 2012.

[13] B. J. Foster, L. McCauley, and R. H. Mak, "Nutrition in infants and very young children with chronic kidney disease," Pediatric Nephrology, vol. 27, no. 9, pp. 1427-1439, 2012.

[14] KDIGO, "KDIGO 2012 clinical practice guideline for the evaluation and management of chronic kidney disease," Kidney International Supplements, vol. 3, no. 1, 2013.

[15] National Kidney Foundation, "K/DOQI clinical practice guidelines for chronic kidney disease: evaluation, classification, and stratification," The American Journal of Kidney Diseases, vol. 39, no. 2, supplement 1, pp. S1-S266, 2002.

[16] North American Pediatric Renal Trials and Collaborative Studies (NAPRTCS) 2011 Annual Dialysis Report. 


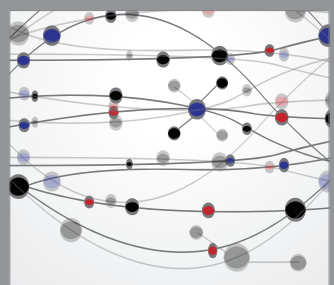

The Scientific World Journal
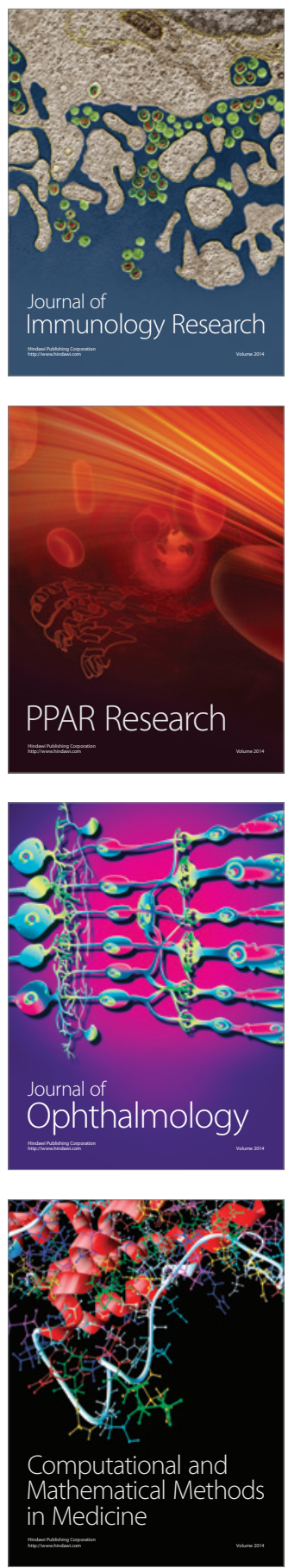

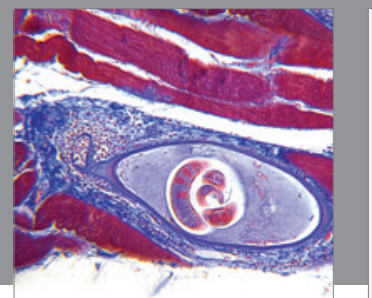

Gastroenterology

Research and Practice
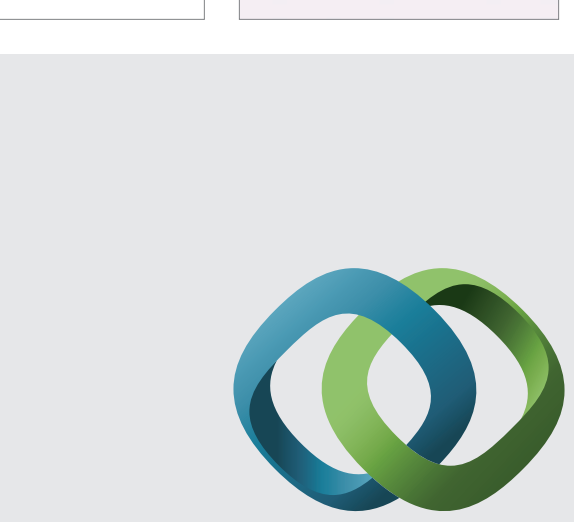

\section{Hindawi}

Submit your manuscripts at

http://www.hindawi.com
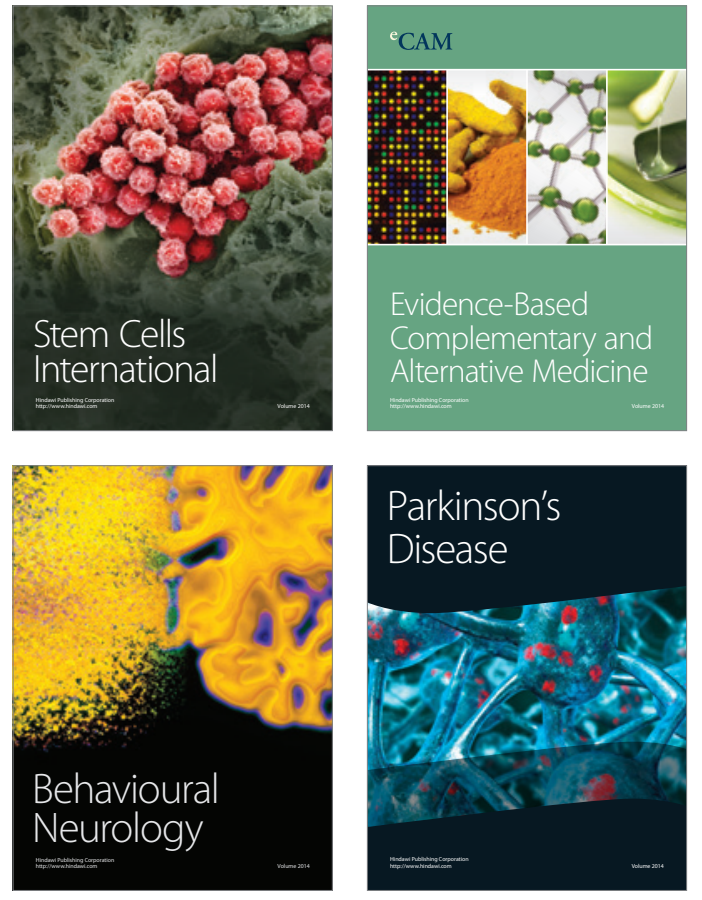
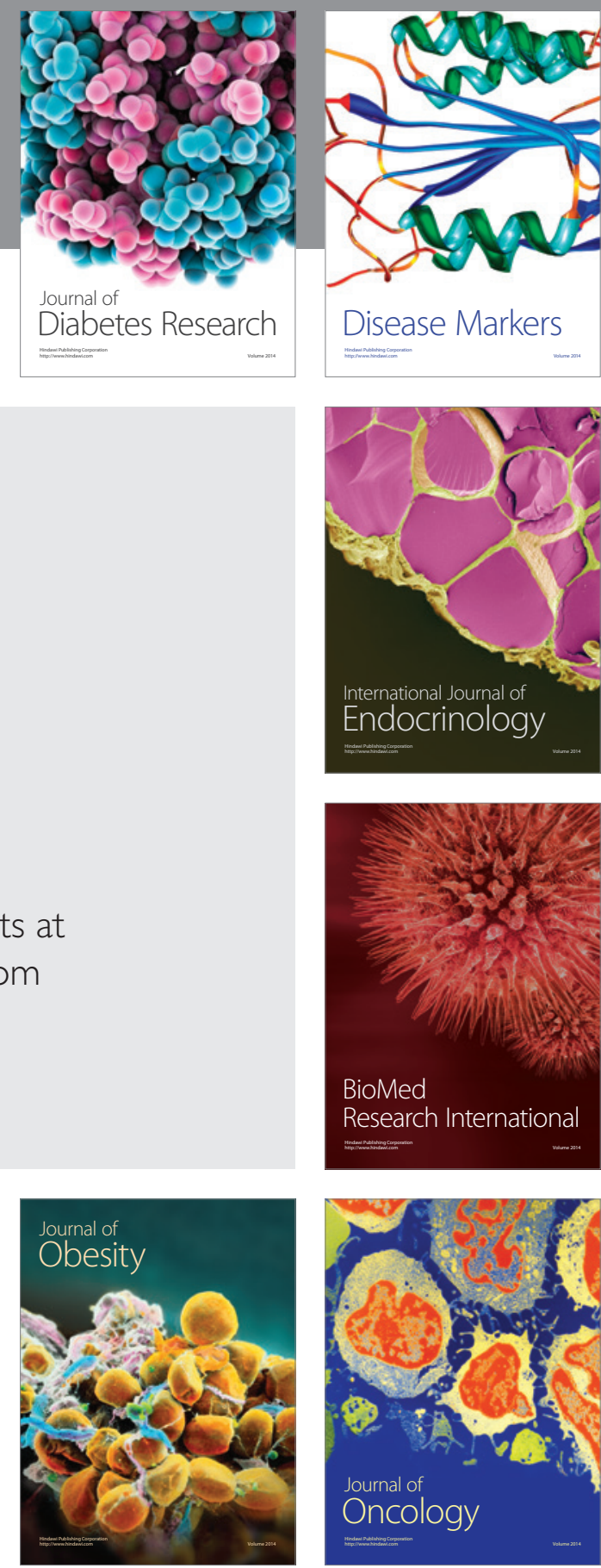

Disease Markers
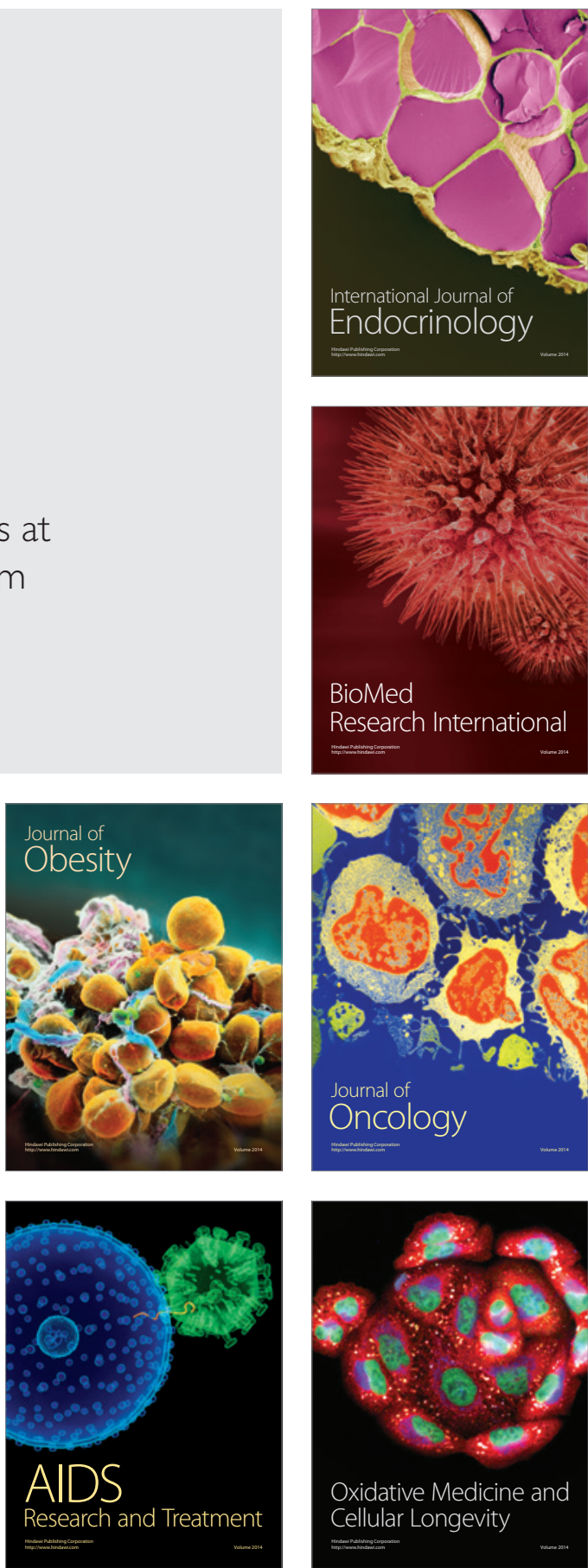